http://dx.doi.org/10.22402/j.rdipycs.unam.3.1.2017.151.13-19

\title{
PRESENTACIÓN DEL NÚMERo
}

\section{| VOLUMEN 3 | NÚMERO 1 | ENERO-JUNIO 2017 |}

\section{TABLA DE CONTENIDO}

INTRODUCCIÓN

ESQUEMA EDITORIAL

ANÁLISIS DE LOS DICTÁMENTES DE LOS REVISORES

panorama General, 14

Perfil de Evaluación entre pares, 15

Calificación Promedio de los Artículos, 16

Índice de Concordancia, 16

Correlograma, 17

Desacuerdo entre revisores, 17

Material Científico Publicado, 18 


\section{INTRODUCCIÓN}

$\mathrm{E}$ ste primer número del 2017 de la Revista Digital Internacional de Psicología y Ciencia Social (RDIPyCS), constituye el tercer volumen de la revista e implica la inquietud del equipo editorial por mantener políticas de publicación científicas acordes a la era de la información, en donde se desarrollen alternativas de difusión incluyentes, innovadoras, transparentes, abiertas y gratuitas.

El volumen anterior enfatizó la invocación de un sentido de responsabilidad social en la ciencia a traves de la construcción de un tejido social científico en donde impere un pluralismo sociológico caracterizado por la aceptación de la diversidad y se realicen acciones de equidad en un entorno transdisciplinar.

En este nuevo número se da continuidad a la búsqueda de mejora, por lo que se incluyen en este número interesantes artículos en distintos formatos de publicación, como lo son: el tradicional artículo en formato PDF, el práctico formato ePub, así como un accesible formato MP3; permitiendo así alcanzar cada vez más lectores y contribuir a la difusión de la psicología como ciencia. Se pretende, por otro lado, ratificar el compromiso con autores, revisores y lectores al garantizar la transparencia de todo el proceso editorial a través de herramientas tales como Open Journal Systems y Content Management Systems.

\section{ESQUEMA EDITORIAL}

En seguimiento con el compromiso adoptado por la revista de transparentar las decisiones científico-editoriales, este primer número del segundo volumen de la RDIPyCS expone a la comunidad científica todos los procesos que utiliza para transferir y difundir la ciencia. Estos procesos se desarrollarán en un esquema editorial que se utiliza en todos los artículos que se publican y consiste en:

- Portada. Está constituida por título, el resumen en español e inglés de artículo, las palabras claves y la forma de citar de acuerdo a las reglas establecidas por el American Psychological Association (APA).

- Bitácora. Conformada por el título del artículo, los tiempos editoriales (cuándo se recibió, cuándo fue aceptado para su publicación y la fecha en que fue publicado); las imágenes iconográficas de los dos primeros autores/as del artículo, en caso de que sean más; las secciones de resúmenes curriculares; las contribuciones que hicieron cada uno de los autores/as en la elaboración del artículo; los agradecimientos y los datos de afiliación de las autores/as.

- Índice. Es una serie de vínculos que permitirán al lector navegar en forma libre por el cuerpo del artículo, lo que evitará una lectura secuencial del material científico publicado.

- Cuerpo. Consiste en el artículo propiamente. Los apartados en que se desarrolle el material científico dependerán del tipo de estudio que se esté reportando. Si desea conocer los que se aceptan en esta revista diríjase al siguiente vínculo.

- Meta-análisis del artículo. Está constituido por procedimientos meta-analíticos en donde se presentan cuantitativamente las opiniones que emitieron los evaluadores sobre el artículo. Esta sección del artículo tiene tres partes: la primera es el perfil en donde se presentan las opiniones que emitieron los revisores en cuanto a la calidad de la introducción, la metodología, los resultados y todos los apartados del artículo. La segunda parte presenta la figura de concordancia que existió entre las opiniones de los revisores, construida utilizando el método de Bland y Altman (1986). Finalmente, la tercera muestra el nivel de acuerdo que se dio entre los revisores al calificar todos los apartados del material científico.

- Historia del proceso editorial. En esta sección hay dos vínculos, uno permite el acceso al meta-análisis en línea, y en el otro se puede consultar todo el proceso editorial del artículo, que incluye las conversaciones entre los autores y los revisores, es decir, el intercambio dialógico entre los actores científicos.

\section{ANÁlisis de LOS DICTÁMENTES DE LOS REVISORES}

\section{Panorama general}

En esta sección se presenta un análisis global de los dictámenes que los revisores hicieron de todos los artículos publicados en este número de la revista. Podría decirse que es una especie de radiografía en conjunto que nos habla de la opinión que los revisores se formaron de los materiales científicos publicados. Con esto se busca que el lector tome como punto de referencia la opinión de los revisores, que son gente de la comunidad científica; la contraste con su opinión y saque sus propias conclusiones sobre la calidad, relevancia e impacto de lo que se publicó en este número.

De esta manera se irá formando una opinión pública fundamentada en la transparencia de las decisiones que se toman para transmitir el conocimiento científico; es decir, una opinión pública informada producto del entendimien- 
to personal que tiene cada revisor/a y de la valoración que hagan los lectores del material científico divulgado.

Al igual que en cada artículo, en este apartado de la revista se presenta el meta-análisis, pero a diferencia de aquel, aquí se muestra el resultado de la opinión que emitieron los revisores/as en su conjunto de los artículos publicados. Las secciones son: el perfil de evaluación entre pares, la calificación promedio otorgada a cada uno de los artículos, el índice de concordancia, el correlograma y el índice de desacuerdo entre revisores/as.

Es importante mencionar que, aunque se habla de los revisores/as, esto no significa que cada uno de ellos haya evaluado todos los artículos publicados en la revista, sino más bien para este meta-análisis se consideró a cada revisor como una unidad de análisis y cada artículo como si fuera uno mismo, es decir, el conjunto de dictámenes se tomó a manera de una unidad replicativa, ya que cada dictamen fue hecho por diferentes revisores/as.

Si bien como lo señalan Glass, Wilson, y Gottman (1975); Yin (1984); Menard (1991) y Silva Rodríguez (2004), los análisis que se basan en unidades replicativas son más propensos a verse afectados por fuentes de invalidez, debido a la alteración de la naturaleza de los individuos (en nuestro caso diferentes revisores/as) que componen la unidad de observación, puesto que es probable confundir el efecto de los juicios de los revisores/ as con cambios abruptos en la composición de dicha unidad. Sin embargo, la unidad replicativa está menos sujeta a efectos de información y reactividad, puesto que si fueran los mismos revisores/es de todos los artículos

$$
\text { Opinión }=\frac{\left(a_{11}+a_{21}\right)+\left(a_{32}+a_{42}\right)+\ldots+\left(a_{1 j}+a_{1 j}\right)}{n}
$$

que se publican en un determinado número de la revista acarrearían su sesgo en todos los artículos evaluados.

Tomando en consideración lo anterior, el algoritmo que se utilizó para calcular el perfil de la revista, los índices de concordancia y acuerdo, así como el correlograma y la calificación otorgada a los artículos de este número fue el siguiente:

En donde:

- a es la opinión de la revisión i del artículo j

- iésima revisión $=1,2, \ldots$, hasta $n, y$

- jésimo artículo $=1,2, \ldots$, hasta $k$

Que para el caso de este número de la revista n es igual a 8 revisores y $\mathrm{k}$ igual a 4 artículos.

\section{Perfil de Evaluación entre pares}

Por su parte el perfil evalúa la apreciación que le asignaron los revisores a las partes más amplias que constituyen el reporte de la literatura científica como son la introducción, el método, los resultados y todos los apartados del artículo.

\section{Introducción}

En la figura 1 se muestra la opinión que emitieron los revisores respecto a la introducción de todos los artículos. Como se puede observar, los revisores consideraron que el conglo-merado de artículos presentan un desarrollo muy satisfactorio en la introducción de cada uno de los artículos, ya que de manera promedio puntuaron 3.02 de cinco puntos posibles. Por otra parte, la definición de variables fue el rubro más cuestionado en el proceso de dictaminación, este aspecto fue considerado como muy deficiente con una puntuación de 1.00.

Esta sección en particular puede ser considerada como satisfactoria ya que mayoría de los elementos de evaluación fueron catalogados en esta categoría, con la excepción de la ya mencionada definición de variables y el rubro de objetivo e hipótesis que se calificó como deficiente. sis fueron calificados dentro de esa categoría.

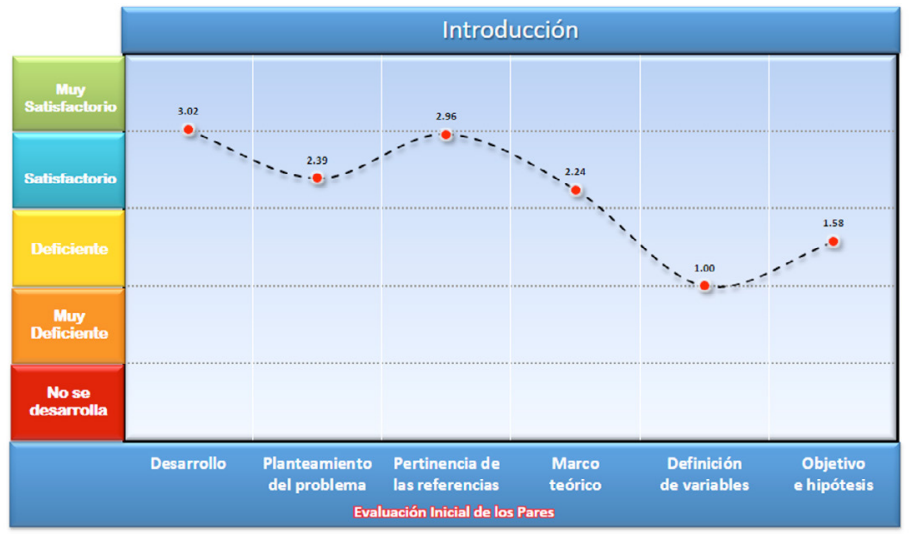

Figura 1.

Perfil de la opinión de los revisores sobre cada uno de los componentes de la introducción de los artículos.

\section{Método}

La sección del método fue la que recibió la calificación más baja en este número al ser catalogada como deficiente en la mayoría de sus elementos y como muy deficiente en cuanto a cuestiones éticas, siendo esta la calificación más baja con una puntuación de 0.56 . Por otra parte, la puntuación más alta entre los elementos del método fue de 1.80 para la recolección de datos, por lo que se considera como deficiente en la evaluación global de los artículos de este número. 


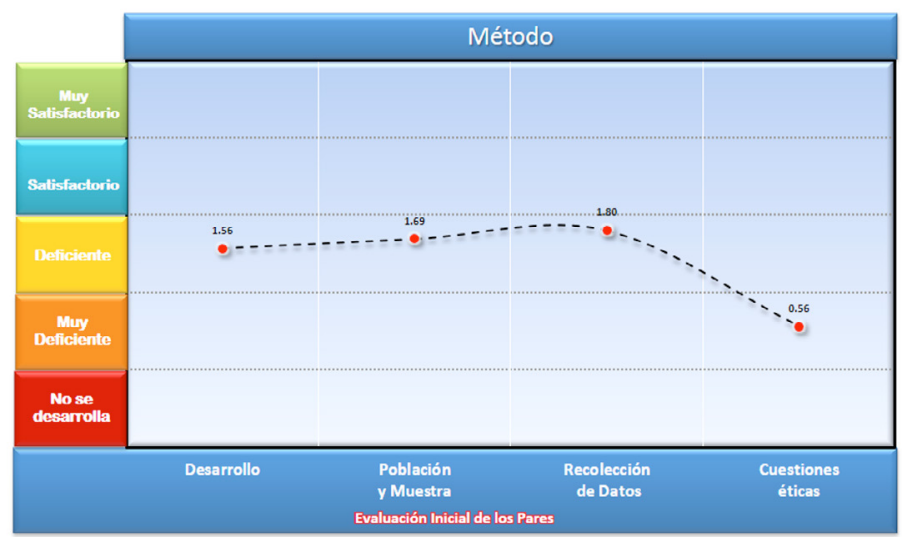

Figura 2.

Perfil de la opinión de los revisores sobre cada uno de los componentes del método de los artículos.

\section{Resultados}

La sección de resultados presenta menor variabilidad que las secciones señaladas pre-viamente; en este apartado se destaca el desarrollo con una puntuación de 2.41, por lo que se le puede considerar satisfactorio. En contraste, el análisis de datos obtuvo la me-nor calificación de la sección con 1.84, siendo catalogado como deficiente. En términos generales este apartado de los artículos presentes en este número fue considerada como deficiente por parte de los dictaminadores.

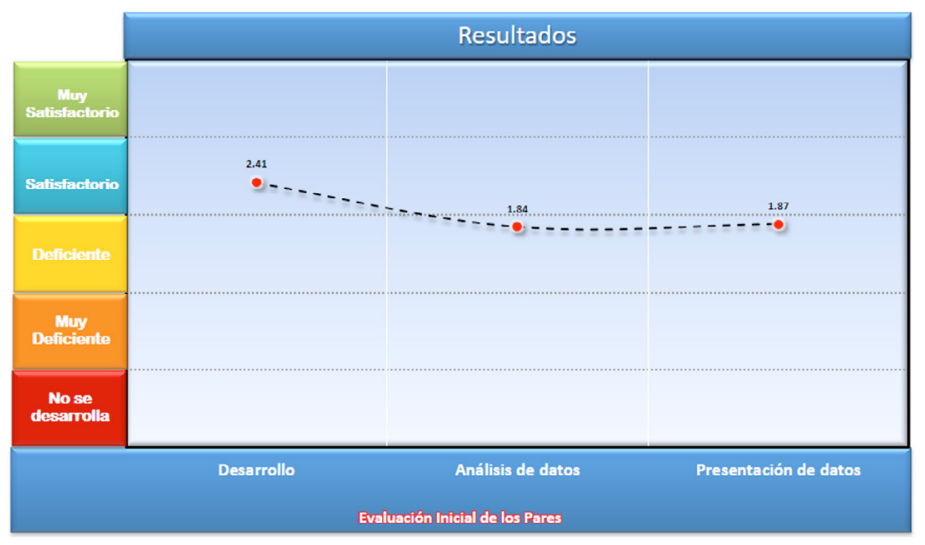

\section{Figura 3.}

Perfil de la opinión de los revisores sobre cada uno de los componentes de los resultados de los artículos.

\section{Todos los apartados}

En la figura 4 se muestra la opinión emitida sobre todos los apartados. Como se puede observar, los revisores otorgaron puntajes que varían de satisfactorio a deficiente, siendo las calificaciones más altas las referentes al título (3.60), la apreciación global de los artículos (3.35) y el resumen (3.22), siendo considerados como muy satisfactorios.
En contraparte, el método obtuvo la calificación más baja siendo la única sección considerada como deficiente. En términos generales se pueden considerar una calificación muy satisfactoria para los artículos de este número, ya que este punto de corte en las calificaciones engloba la mayoría de los aspectos evaluados.

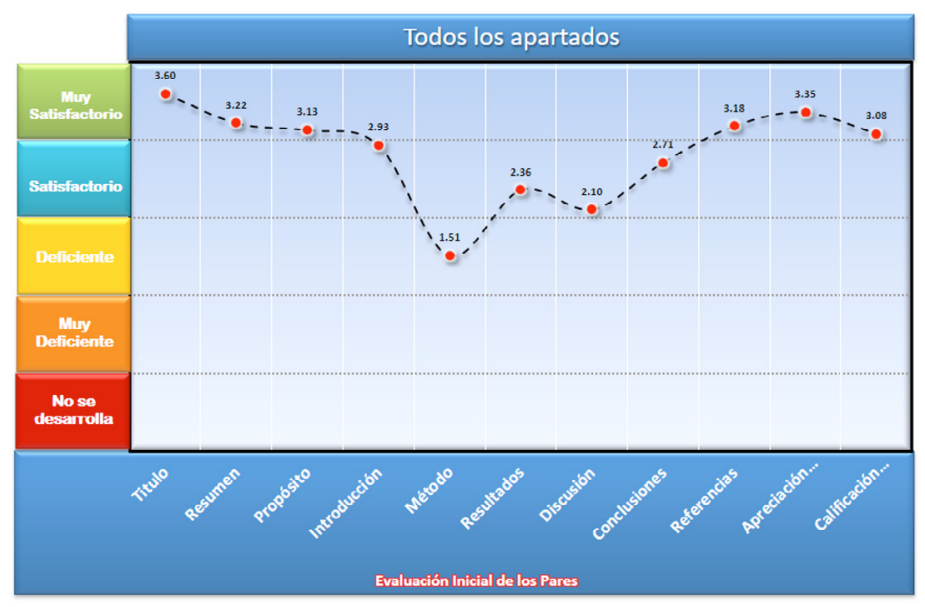

Figura 4.

Perfil de la opinión de los revisores en todos los apartados de los artículos.

\section{Calificación Promedio de los Artículos}

En la figura 5 se presenta la calificación promedio otorgada por los evaluadores al artículo que revisaron. En dicha figura puede observarse que la calificación otorgada a los artículos varió en una escala del 0 al 100 entre 95.17 y 57.47, que corresponde al artículo escrito por los autores Guarneros, Maldonado y Silva, y al escrito por Rodríguez, Cruz, Leija y Domínguez respectivamente.

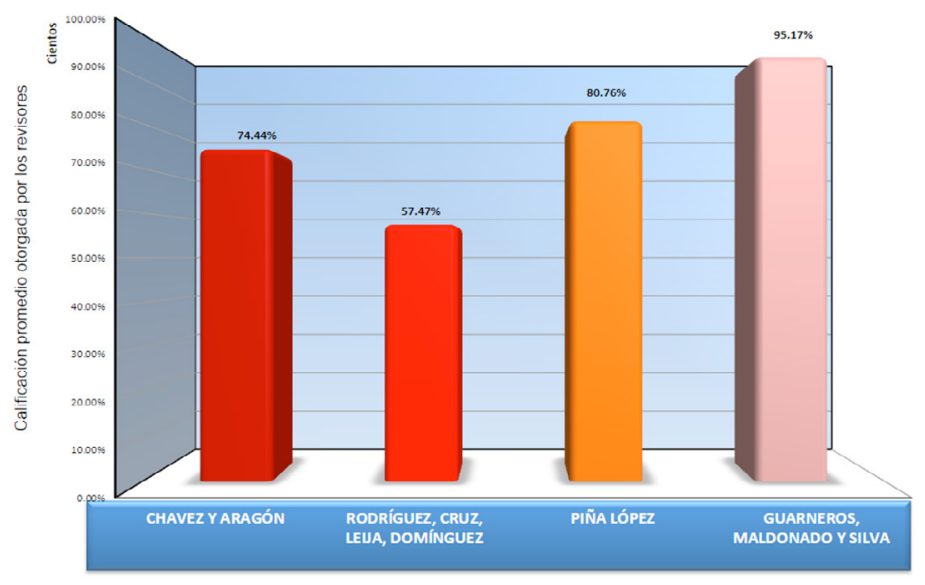

Figura 5.

Calificación promedio otorgada por los revisores los artículos.

Como se puede observar las puntuaciones fluctúan en un rango de $385 \%$ aproximadamente, presentando 
una alta variabilidad; el artículo de Chávez y Aragón obtuvo una puntuación de 74.44, mientras que el del autor Piña-López alcanzó una calificación de 80.76.

\section{Índice de Concordancia}

Este índice indica el acercamiento que hubo en los juicios de los revisores; es decir, la magnitud en que éstos reportan resultados iguales; mide por lo tanto la variabilidad entre sus opiniones o juicios con respecto a los dictámenes que emitieron del artículo.

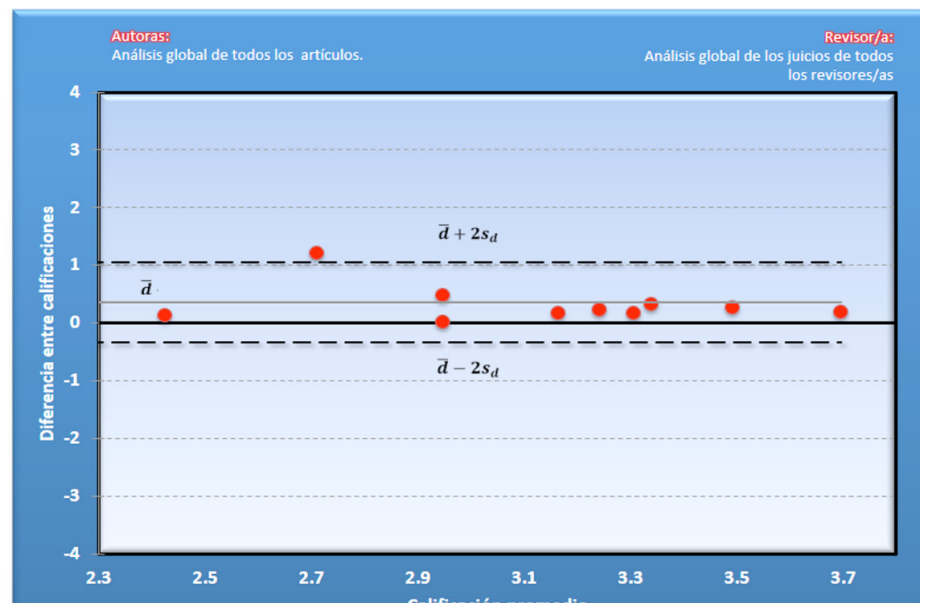

Figura 6.

Indice de concordancia en los juicios de los revisores.

Para construir la figura 6, se tomó la calificación promedio de los juicios que los revisores asignaron a las nueve partes del artículo, como fueron título, resumen, propósito, introducción, método, resultados, discusión, conclusiones y referencias.

En dicha figura se observa que la diferencia fue significativa, ya que la superó el límite de las desviaciones estándar siendo el límite inferior 0 y el superior fue mayor a 1.

\section{Correlograma}

En la figura 7 se muestra el correlograma de los juicios que emitieron los revisores, es una figura construida a partir de calcular los índices de correlación entre las opiniones emitidas. Los juicios de los revisores que se correlacionaron en el mismo sentido y con una magnitud moderada fueron los que revisaron el artículo de Piña-López con un índice de 0.73, después el de Chávez y Aragón con 0.71; seguido del artículo escrito Rodríguez, Cruz, Leija y Domínguez (0.58). Las opiniones de los revisores que no se correlacionaron fueron los que dictaminaron el artículo de Guarneros, Maldonado y Silva con una puntuación de 0.09 .

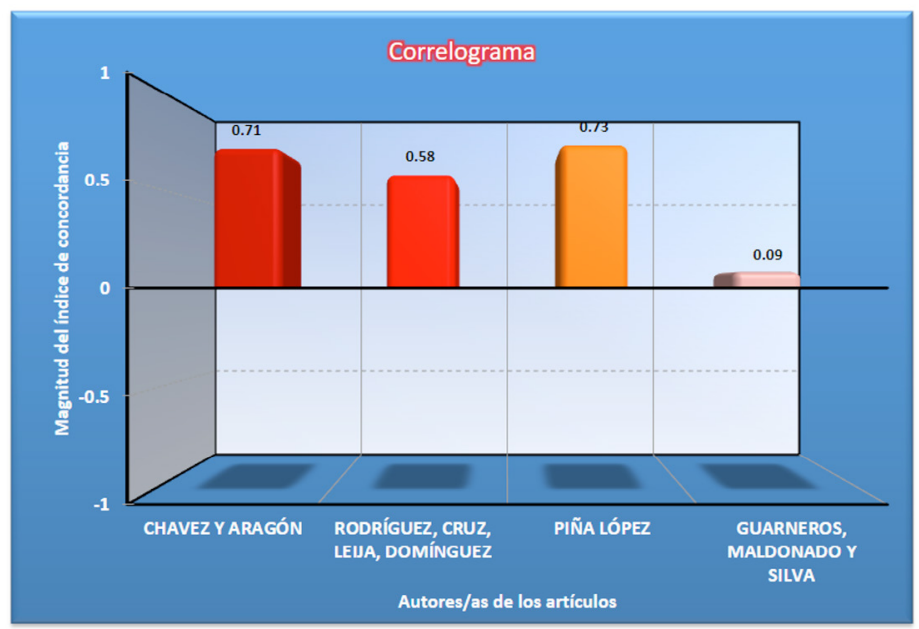

Figura 7.

Correlación entre los juicios de revisores.

\section{Desacuerdo entre revisores}

Finalmente, la figura 8 muestra el desacuerdo entre los revisores, independientemente de su sentido, puesto que sólo evalúa la magnitud de la diferencia de opinión.

Los revisores que menos desacuerdos tuvieron fueron los que dictaminaron el artículo de Rodríguez, Cruz, Leija y Domínguez al tener menos de un punto entre ambas calificacio-nes (57.60 y 57.35). El artículo que presenta una mayor diferencia en su dictaminación fue el escrito por Chávez y Aragón con una diferencia de 35.72, recibiendo calificaciones de 92.30 y 56.58 .

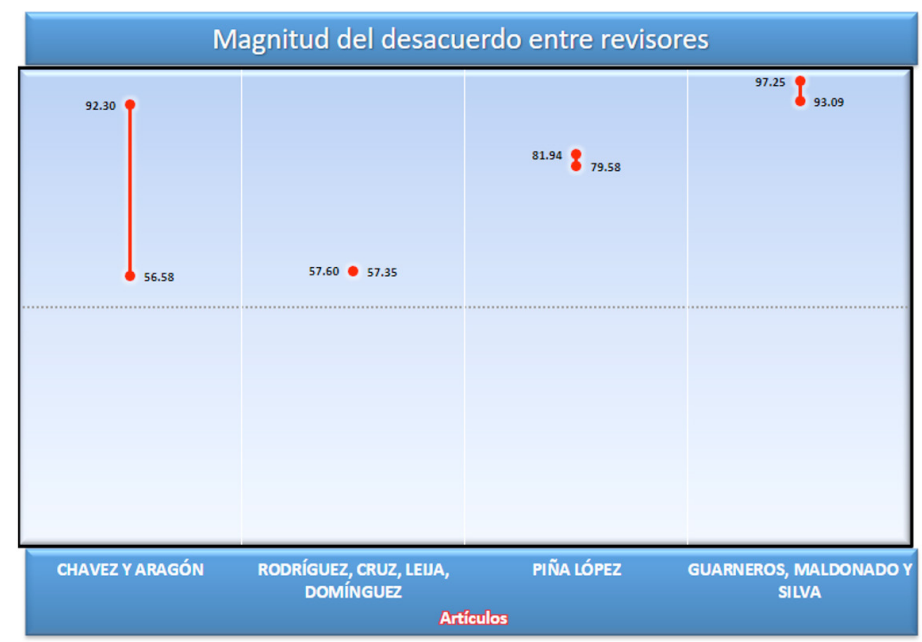

Figura 8.

Desacuerdo entre revisores.

Estas discrepancias en la apreciación de la calidad de los artículos son valoraciones que los revisores las perciben subjetivamente, lo relevante de todo esto es encontrar el punto en donde las diversas subjetividades, 
principalmente de los revisores, coincidan para así tener una revista que facilite la comunicación científica, no obstante que sea una realidad artificial producto de los múltiples significados y sentidos que los revisores le asignen al material científico revisado.

\section{Material Científico Publicado}

El primer artículo de este número fue escrito por las autoras Chávez y Aragón, en él se busca mostrar la presencia de conductas de bullying en una muestra de 416 estudiantes desde 5o. año de primaria hasta 1o. de preparatoria, así como el índice con que reportan ejecutar habilidades sociales relacionadas de manera primordial como protectoras de bullying, para lo cual se aplicó a dicha muestra la "Escala de habilidades sociales y bullying". Este estudio enfatiza el problema que representa el bullying en México, ya que de acuerdo con especialistas en violencia escolar, México ocupa el primer lugar a nivel internacional en acoso escolar; a su vez, se destaca el reconocimiento a las habilidades sociales como un factor de protección contra este problema.

Los resultados encontrados por las autoras revelan que entre 12 y $47 \%$ cometen conductas de bullying, entre 9 a $18.5 \%$ de ciberbullying y de 20 a $87 \%$ no presentan de manera adecuada habilidades sociales que pueden funcionar como protectoras contra el bullying; encontramos además diferencias significativas en la presencia de bullying y ciberbullying a favor de los hombres y de los alumnos de 5o. de primaria y 2o. de secundaria.

En el artículo escrito por Rodríguez, Cruz, Leija y Domínguez se presenta un estudio piloto cuyo objetivo fue explorar la relación entre la presión arterial media (PAM) y el estrés psicológico en un grupo de participantes clínicamente sanos (alimentación, conductas preventivas y valoración negativa ante eventos estresantes dentro de rangos normales). Para valorar su nivel de estrés, incluyendo los criterios de inclusión, los autores utilizaron el Perfil de Estrés de Nowack.

Posteriormente administraron a los participantes el Trier Social Stress Test (TSST) para explorar el efecto de este paradigma sobre la PAM. Los resultados mostraron una relación directa estadísticamente significativa entre la PAM y el nivel de estrés, y una relación inversa entre la PAM y el apoyo social. La PAM post TSST aumentó estadísticamente. Los resultados se analizan en el contexto de la utilidad de estas medidas clínicas para diseñar programas de intervención basados en evidencia y necesidades específicas con aplicaciones inmediatas en contextos universitarios para manejo de estrés.

El tercer artículo del presente número fue elaborado por el investigador independiente Julio Alfonso Piña.
El autor presenta un artículo de corte teórico sobre la psicología positiva (PP) como un Ilamado divino al que respondió Seligman, el cual ha traído consigo la postulación de dogmas de fe defendidos a ultranza por una legión de fieles devotos. Un Ilamado que se justifica en un juego de lenguaje particular y en creencias que, por prescripción o dogma (de fe), se nos dice tenemos que aceptar. En este trabajo se examina cómo el juego de lenguaje en la PP y las creencias que se le desprenden se justifican en la razón natural, no la científica, concluyéndose que la PP es en última instancia una propuesta que se enmarca en la conocida como psicología folk.

En la artículo escrito por Guarneros, Maldonado y Silva se habla sobre la existencia de laboratorios de investigación virtuales, los cuales permiten comprobar teorías con demostraciones virtuales. En la enseñanza es necesaria la práctica y simulación de aprendizajes que requieren los profesionales de psicología para el ámbito aplicado, de este escenario, el objetivo de artículo fue diseñar un laboratorio virtual en psicología a través de un diseño instruccional basado en el Aprendizaje Basado en Problemas ABP, para que los estudiantes de psicología desarrollen habilidades y amplíen su repertorio de competencias, para sobre cómo evoluciona el lenguaje y problemas del habla como dislalias. para estudiantes de psicología de sistema en línea de la UNAM y profesionales relacionados al ámbito infantil. Se describe el proceso de diseño y se presentan resultados sobre el funcionamiento del laboratorio virtual en un grupo piloto de 203 participantes. Los resultados demuestran que es un entorno de aprendizaje simulado el cual pone en práctica habilidades que desarrolla el ABP.

Finalmente se presenta una traducción elaborada por Delabra del escrito "Conduct of every life. Implications for critical psychology" escrito por Ole Drier. En el escrito se desarrolla la idea de que las personas no están meramente localizadas en una situación estática. Su situación inmediata es una parte particular de su vida cotidiana en curso. Y el significado de una situación y cómo una persona se involucra en ella, depende de qué parte forma de su vida cotidiana. El sustento de la subjetividad y la experiencia es la vida cotidiana de las personas y no una situación. Por tanto, el concepto de conducción de la vida cotidiana tiene un potencial importante para la psicología, sobre todo porque se trata de una herramienta que permite capturar la subjetividad humana desde el punto de vista de dónde y cómo viven los sujetos sus vidas cotidianas en estructuras de práctica social. En este escrito se exponen las principales razones por las que este concepto se ha integrado en el marco teórico de la psicología crítica y, además, se 
abordan dimensiones importantes del mismo y algunos retos sobresalientes que plantea no sólo a la psicología crítica, sino a la psicología en general.

\section{Referencias}

Bland, J. M., \& Altman, D. G. (1986). Statistical methods for assessing agreement between two methods of clinical measurement. Lancet, 327(8), 307-310.

https://wwwusers.york.ac.uk/ mb55/meas/ba.pdf
Glass, G. V., Wilson, F., \& Gottman, G. (1975). Design and analysis of time-series experiments. Boulder: Colorado Associated Press.

Menard, S. (1991). Longitudinal research. London: SAGE University paper.

Silva Rodríguez, A. (2004). Diseño de investigación con $\mathrm{N}=1$ o series de tiempo. In A. Silva Rodríguez (Ed.), Métodos cuantitativos en psicología. Un enfoque metodológico (pp. 45-70). México: Trillas.

Yin, R. K. (1984). Case study research: design and methods. Thousand Oaks: Sage Publications, Inc.

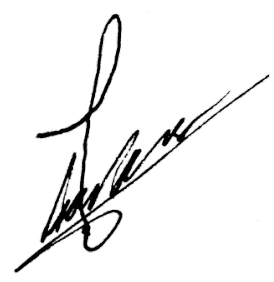

Oscar Giovanni Balderas Trejo Diseñador editorial 